\title{
Eleven-year solar cycle periodicity in sky brightness observed at Norikura, Japan
}

\author{
Takashi Sakurai \\ National Astronomical Observatory, Mitaka, Tokyo 181-8588, Japan \\ (Received December 15, 2000; Revised October 30, 2001; Accepted October 30, 2001)
}

\begin{abstract}
We analyzed the brightness of the sky background observed with a coronagraph at Norikura, Japan, in the period of 1951-1997. We discovered that the power spectrum shows a clear eleven-year periodicity of solar activity cycle, in addition to strong annual variations. The peaks in the eleven-year component are found in the declining phase of activity, 2-4 years after the sunspot number maximum. The brightness of the continuum corona is far fainter than the observed amplitude of the eleven-year component and cannot account for the observed phenomenon. A possible interpretation is that the solar activity modulates the contents of aerosols in the upper atmosphere of the earth, thus producing variations in scattering of sunlight. Why this effect is largest in the declining phase of solar activity is still unknown.
\end{abstract}

\section{Introduction}

The transparency of the sky has been measured in several ways; stellar extinction observations (Thompson and Lockwood, 1996), lidar backscatter measurements (Barnes and Hofmann, 1997), solar photometer measurements (Dutton et al., 1994), and satellite-based solar extinction observations (Long and Stowe, 1994). These authors studied the relation between sky transparency and volcanic activities. An extensive review is found in Sato et al. (1993).

Here we present the measurements of the sky brightness just next to the sun by a coronagraph. A similar approach was also taken by LaBonte (1999), using the data taken at Haleakala, Hawaii, in the period of 1955 through 1998. The observations were not performed or were infrequent in 1957-1966, 1974-1979, and 1983-1986. His study was mainly on the sky brightness changes caused by volcanic eruptions. Our main interest here is in periodicities in the sky brightness. We discovered that the sky brightness shows an eleven-year solar cycle modulation.

The coronagraph, invented by B. Lyot in 1930 (Lyot, 1939), is a telescope specifically designed to observe the solar corona. At the primary image of the sun, an occulting disk is placed and blocks the light from the main disk of the sun (the photosphere). The image outside the occulting disk is relayed to the final image plane and forms the image of the corona.

At the wavelength range near $5300 \AA$ where our observations are carried out, the brightness of the sky is a few tens of $10^{-6} I_{0}$ (van de Hulst, 1953), where $I_{0}$ means a spectral intensity of the solar disk center at around $5300 \AA$. The brightness of the corona in continuum (the so-called $\mathrm{K}$-corona) is about $10^{-6} I_{0}$, and is much fainter than the sky. Usually the coronagraph employs a spectrograph (or a narrow band filter) to isolate some of emission lines. The coronal emission

Copy right(C) The Society of Geomagnetism and Earth, Planetary and Space Sciences (SGEPSS); The Seismological Society of Japan; The Volcanological Society of Japan; The Geodetic Society of Japan; The Japanese Society for Planetary Sciences. lines, e.g. Fe XIV $5303 \AA$, have an intensity of a few tens of $10^{-6} I_{0}$ and can stand up above the level of the sky background. In order to measure the strength of the emission line, it is a customary procedure to measure the continuum level just next to the wavelength of the emission line, and to take the difference to obtain the net strength of the emission line. As a bi-product the brightness of the sky next to the solar disk is recorded. This sky background brightness is mostly due to the scattering of sunlight by dust, water, and ice particles (or aerosols) in the atmosphere. (Rayleigh scattering due to air molecules gives rise to about $10^{-6} I_{0}$ at 5000 A.) The scattered light thus formed is again scattered or absorbed by these particles, but this is a minor effect compared to the supply of photons from the sun by scattering. Therefore, increase in the population of scatterers means increase in sky brightness.

\section{Observatory and Instrument}

The Norikura Solar Observatory, National Astronomical Observatory of Japan, was established in 1950. The observatory is located in Northern Japan Alps, $137^{\circ} 33^{\prime} \mathrm{E}, 36^{\circ} 07^{\prime} \mathrm{N}$, at an altitude of $2876 \mathrm{~m}$. Regular measurements of the coronal emission line of Fe XIV at $5303 \AA$ (the so-called 'coronal green line') started in 1951. Instrumental details and calibration procedures were described in Sakurai et al. (1999). Briefly summarizing, the coronagraph of classical Lyot's design has an effective aperture of $10 \mathrm{~cm}$ and a focal length of $150 \mathrm{~cm}$. The occulting disk blocks the sun at a height of $35^{\prime \prime}$ above the limb. The final image scale is adjusted to $100^{\prime \prime} / \mathrm{mm}$ (solar diameter roughly $19 \mathrm{~mm}$ ) by a set of relay lenses. At the final image plane is placed a slit of $9 \mathrm{~mm}$ in length. Behind the slit is equipped a spectroscope made of three blocks of prisms, with a linear dispersion of $40 \AA / \mathrm{mm}$ at the green line. The spectrum over a range of $200 \AA$ can be viewed through an eyepiece, and the intensities of the green line and the continuum (sky background) are measured visually, with respect to the reference light source attenuated 
(a)

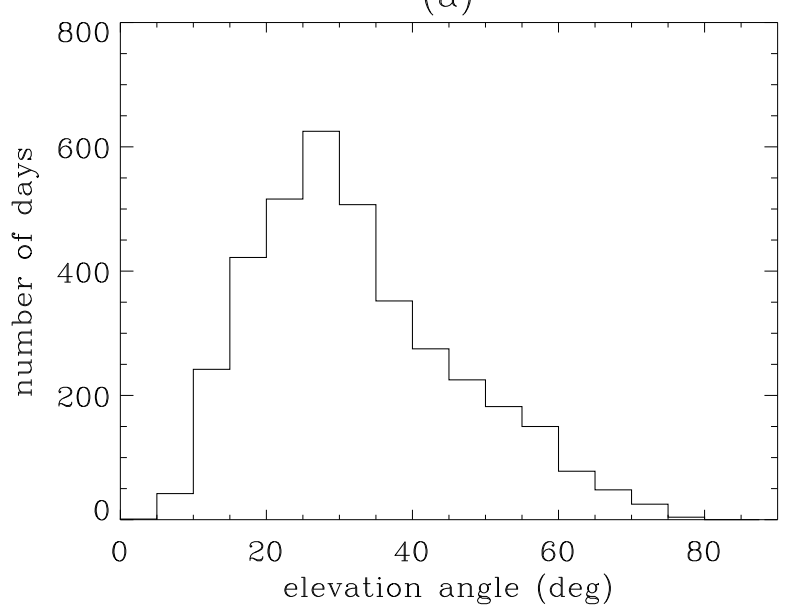

(b)

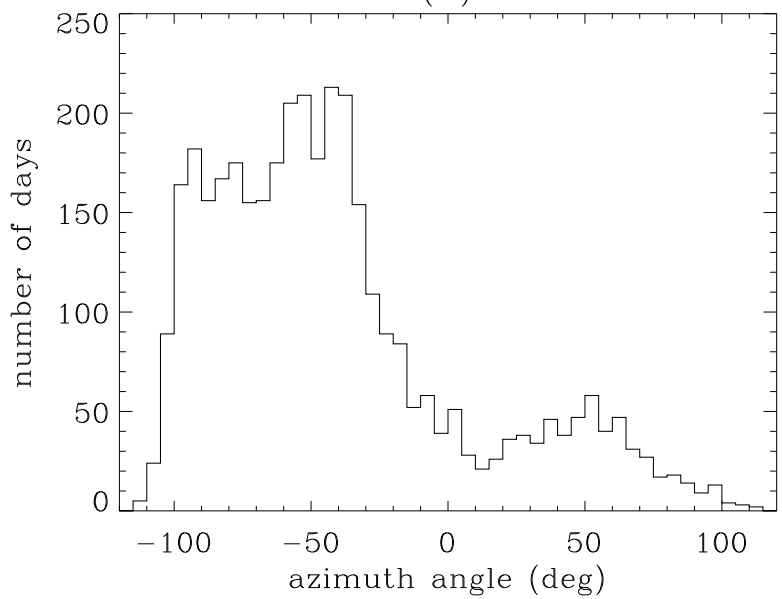

Fig. 1. The distribution of (a) the elevation angles and (b) the azimuthal angles at the time of observation. The azimuthal angle here is defined as zero toward south, and negative (positive) in the eastern (western) directions, respectively. The bin size in the abscissa is 5 degrees.

(a)

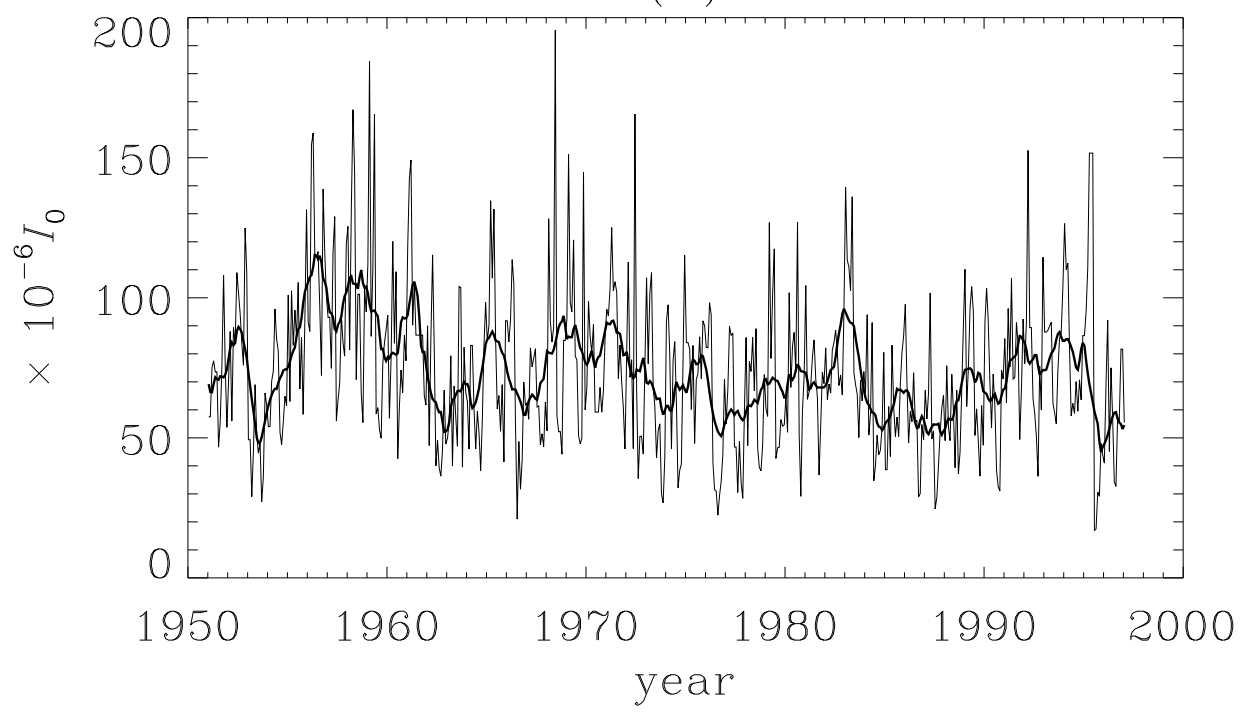

(b)

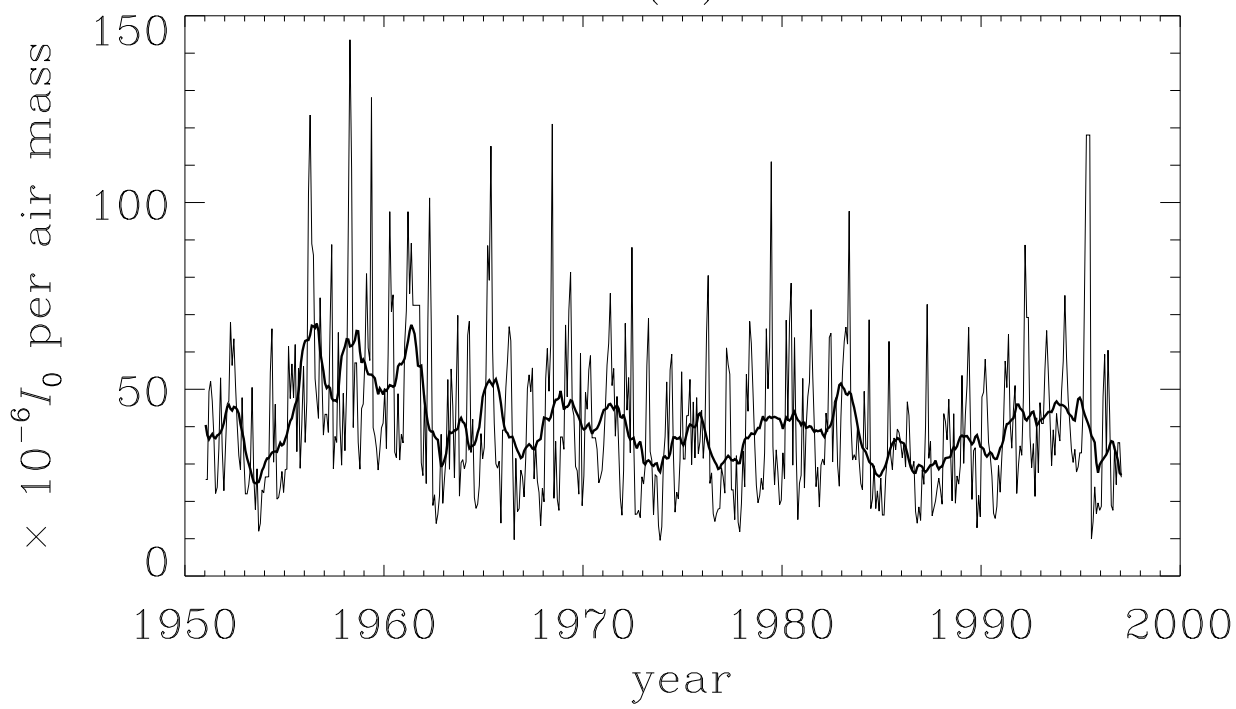

Fig. 2. The brightness of the sky observed at Norikura, Japan, in the period of 1951-1997; (a) raw data and (b) the data reduced to unit air mass. The thin line shows monthly data, and the thick line is a one-year running mean. 
by a calibrated wedge. Although the slit extends $900^{\prime \prime}$ on the sky, only the intensity at the center of the slit (i.e. at the point closest to the sun) is read out. The original reference light source was a thallium lamp, whose emission line at $5350 \AA$ is similar in color to the coronal green line. In 1981, the standard for photometry has been changed to the disk center light fed through an optical fiber. The accuracy in the measurement is estimated to be $\pm 3 \times 10^{-6} I_{0}$.

In each measurement, a trained observer first measures the sky brightness at $50^{\prime \prime}$ above the east limb, and then measures the intensity of the green line around the solar perimeter at a height of $50^{\prime \prime}$, with 5 degree steps in position angle. The data presented in this paper are the brightness of the sky $50^{\prime \prime}$ above the sun's limb in the direction of celestial east. The corresponding heliographic latitudes of the observed point vary between $-26^{\circ}$ (in April) and $+26^{\circ}$ (in October).

The data from 1951 to 1997 were obtained with the same instrumental setup. In 1997 the system was upgraded to a two-dimensional imaging instrument and has become automated (Ichimoto et al., 1999). The focal-plane optics were newly built, and we must admit that the continuity of data series is lost.

\section{Data Analysis}

Figure 1 shows the histograms of the altitude and azimuthal angles of the sun at the time of observation. The observations were usually done in the early morning. The mean elevation angle is $32^{\circ}$, and the azimuthal angles show a peak in the east. In winter the elevation angle is systematically lower.

The reduction of the raw data to the values per unit air mass has been made by assuming that the air mass is given as $m=1 / \cos$ (zenith angle). This is a crude treatment when the elevation angle is small. We also assume that the sun and the nearby sky are subjected to the same degree of extinction.

The brightness of the sky observed in the period of 19511997 is shown in Fig. 2. There are 22 months in which no data were taken. The largest gap is in 1961 for four months. Two-month gaps are in 1970 and 1995. The rest are single-month gaps. The data in the gaps were interpolated. Figure 2(a) shows the raw data (montly means) and a smooth curve by applying a one-year running mean. Figure 2(b) represents the values per unit air mass (monthly means and a one-year running mean). The mean value of the air mass is 2.16 , and the ratio between the two curves is close to the value of the mean air mass.

The average value of the sky brightness per unit air mass is $40 \times 10^{-6} I_{0}$. The values look higher in the initial ten years, but this can be due to a drift in the calibration scale (Sakurai et al., 1999). Since 1962, no apparent long-term trend is seen. Pollution of air by the increase in traffic is not detected within our measurement accuracy.

Several peaks in the data can be identified with major volcanic eruptions; e.g., El Chichon in 1982 April and Pinatubo in 1991 June. The effects of these eruptions lasted for about a year.

Figure 3 shows the power spectrum derived in two methods; (a) fast-Fourier transform (FFT) and (b) maximum en-

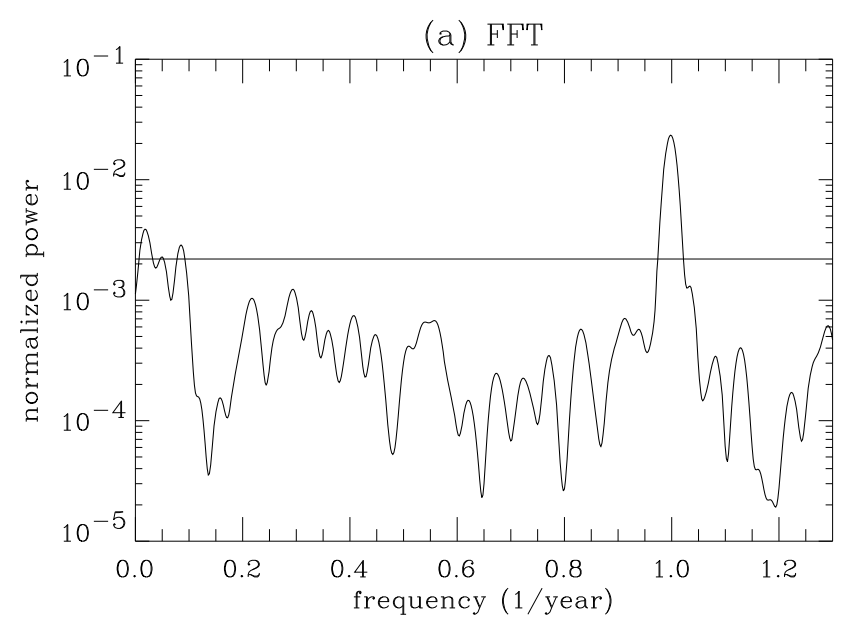

(b) MEM

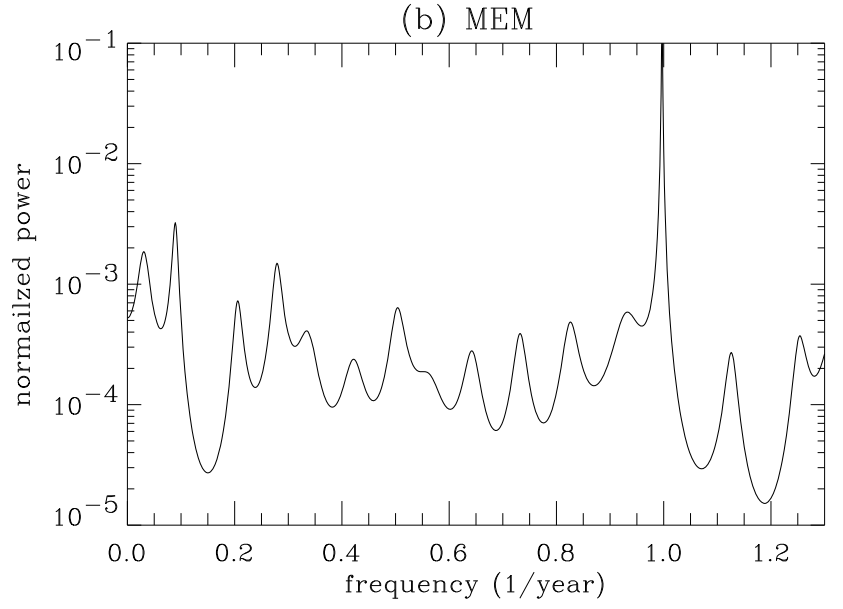

Fig. 3. The power spectra of the sky brightness (a) by FFT and (b) by MEM methods. In panel (a) the $5 \%$ rejection level of white noise is shown as a horizontal line.

tropy method (MEM) (Ulrych and Bishop, 1975). In the FFT method, first we subtracted the average from the data, and then applied a cosine taper on $10 \%$ of data at the ends. Then the data were extended by supplementing zeros. The final bin size of frequency was $1 / 250$ year $^{-1}$. In the MEM method, the maximum lag is taken as 160 months, which corresponded to a local minimum in the final prediction error (FPE).

In both spectra, the highest peak is the annual component. Figure 4 shows the data superposed with a period of one year. Here we found that the sky brightness is highest in spring, probably because scattering by dust from the China continent. In this period the wind is mostly from China toward Japan (north-westerly wind). In summer, because of strong high pressure systems over the Pacific, the wind is southerly from the ocean. A similar annual variation was found in the optical thickness of aerosols above Japan (Husar et al., 1997).

We can also notice a significant peak around eleven years. From FFT and MEM we obtain a period of 11.8 and 11.2 years, respectively. These peaks are presumably related to the solar activity cycle. (The sunspot relative numbers show a period of 10.8 years in the same time period.) For the FFT power spectrum (Fig. 3(a)), the 5\% rejection level 


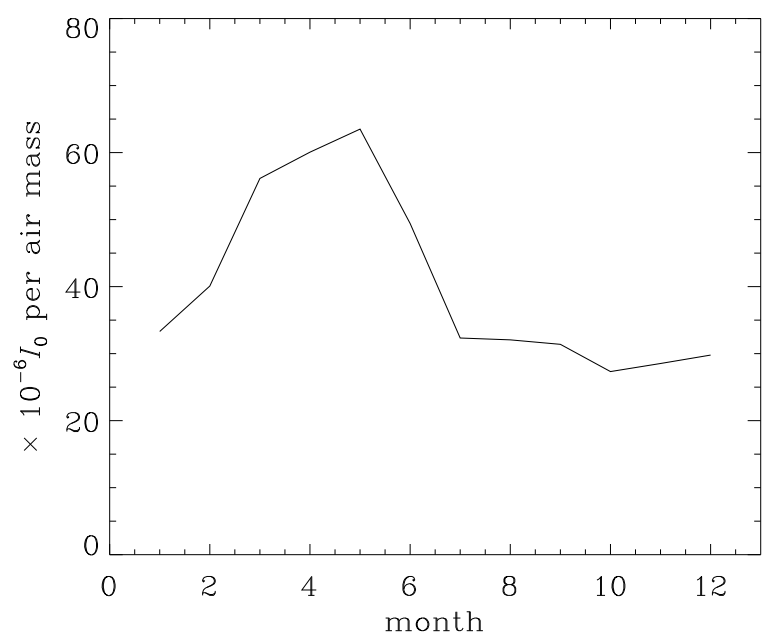

Fig. 4. One year superposition of the data from January (1) through December (12), showing a seasonal variation.

of white noise is shown as a horizontal line (Percival and Walden, 1993). The total power above this line is 0.28 , while from white noise it is expected to be less than 0.003 . The probability of having a peak above this line is therefore less than $5 \%$.

The length of data (47 years) is not sufficiently longer than the expected period of eleven years. The lowestfrequency peak of 0.018 year $^{-1}$ (55-year period) in the FFT spectrum is, therefore, not properly treated by the Fourier method. It is known that MEM can give superior resolution even under such circumstances. The eleven-year peak is more clearly seen in Fig. 3(b) compared to Fig. 3(a), and the lowest-frequency peak is smaller than the eleven-year peak. There are other, shorter periodicities of 2-5 years, but they are less significant compared to the eleven-year component. In terms of atmospheric science, these peaks might bear useful information.

The presence of eleven-year periodicity is very surprising because we have been measuring the sky and not the sun. In order to see the phase relationship with this sky brightness variation and solar activity, we folded the data (the running average curve of Fig. 2(b)) by the solar cycle phase. For simplicity we assumed a period of eleven years, and the data of periods 1953-1964, 1964-1975, 1975-1986, and 19861997 were superposed. The actual sunspot minima are in 1954, 1964, 1976, and 1986, respectively. The results are shown in Fig. 5(a), where the abscissa starts from sunspot number minimum (with an accuracy of one year). In this format, the sunspot maxima are located at year 3-5. The peak in brightness takes place in the declining phase of activity cycle, 2-4 years after the maximum of sunspot relative numbers. The amplitude of variation is about $\pm 8 \times 10^{-6} I_{0}$, which is about $25 \%$ of the mean sky brightness.

The two major volcanic eruptions (El Chichon in 1982 and Pinatubo in 1991) are separated by roughly ten years, and one may suspect that the eleven-year periodicity might be caused by these. However, by suppressing artificially the effects of these known volcanic activities, we still basically obtain the same power spectrum with the eleven-year peak above confidence level. In Fig. 5(a), the effect of El Chi-
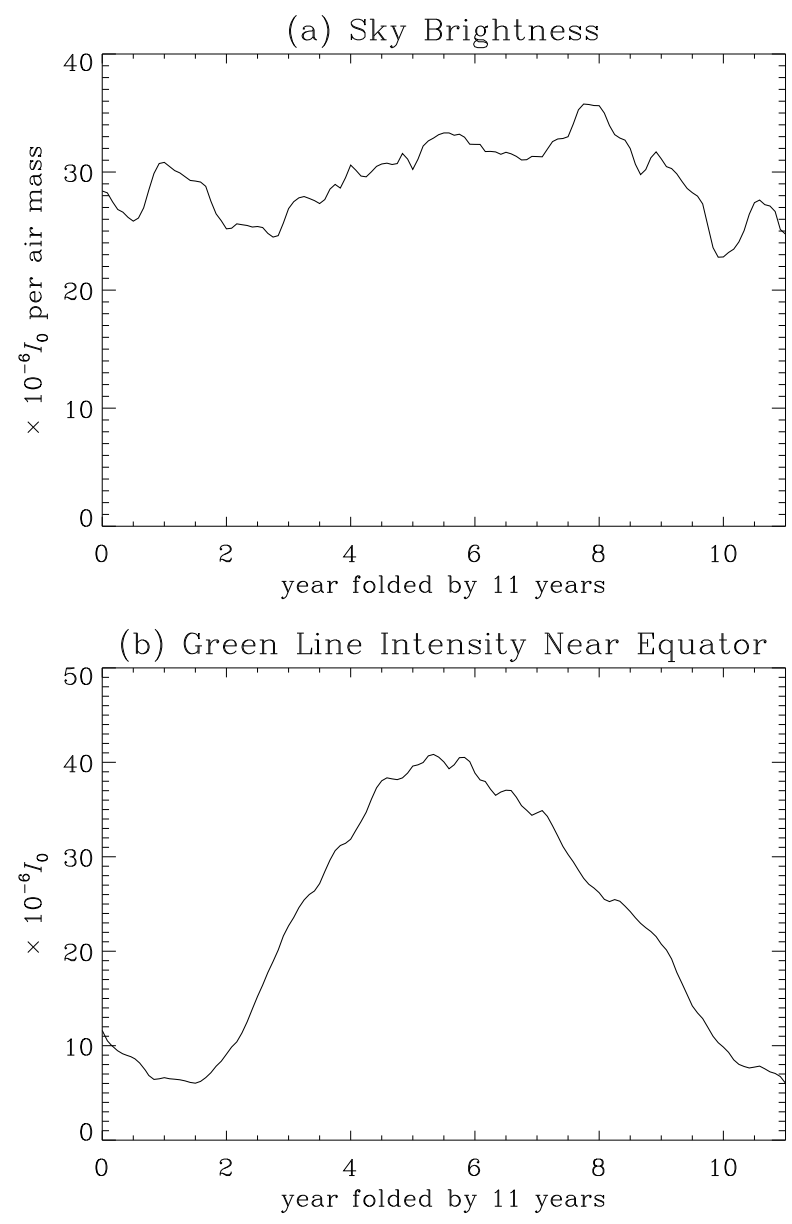

Fig. 5. (a) Eleven-year superposition of the data starting at sunspot minimum, showing a persistent solar cycle modulation of the sky brightness. (b) Eleven-year superposition of the intensity of coronal green line in an equatorial latitude zone (within $\pm 25^{\circ}$ ).

chon explosion in 1982 and Pinatubo explosion in 1991 are located near year 8 and 5.5, respectively. After suppressing the contributions from these eruptions, the peaks at years 5.5 and 8 reduce in height, but the general enhancement after the activity maximum (years 5-9) remains. However, our conclusion is based on the data over four activity cycles, which may not be long enough to completely rule out the possibility that the post-activity-maximum enhancement in sky brightness is due to random superposition of contributions from volcanic eruptions.

\section{Discussion}

We can exclude the coronal origin of this eleven-year periodicity. If the 'sky brightness' we measure is really the solar corona (the K-corona) and not the earth's sky, the amplitude should be smaller than $1 \times 10^{-6} I_{0}$, because the Kcorona itself is at this brightness level (van de Hulst, 1953). The green emission line is safely excluded from the measurement by the usage of the spectroscope, and the variation of the green line intensity is different from Fig. 5(a). Figure 5(b) shows the intensity of the green line in the equatorial latitudes (within $\pm 25^{\circ}$ ) for the same period as in Fig. 5(a). We can see that the green line intensity peaks at the activity maximum. 
Therefore, the remaining possibility is that the solar activity cycle somehow modulates the contents of the scatterers. Since solar-driven changes are larger in the higher layers, we may attribute it to aerosols in the upper atmosphere, which themselves scatter sunlight and also act as nucleation centers for droplets. The variance of the sky brightness changes is as large as $25 \%$, so that the corresponding changes in the solar driver may have to have a similar amplitude. (However, linearity between the driver and the aerosol contents is not at all clear.) The solar far ultraviolet flux (in the wavelength range of 120-200 nm), which reaches down to the upper stratosphere, varies by about $20-30 \%$ between the maximum and the minimum of the activity cycle (Lean, 1987), and can be one of the candidates. The 2-4 year delay of the peak in the eleven-year component of the sky brightness with respect to the sunspot number may be related to the circulation time scale of the earth's atmosphere. Although the detail of the interconnections between solar changes and sky brightness variations is unknown, the data are unique in that the identical instrument has been used for about fifty years to monitor the sky brightness. Our data present one of the most evident solar cycle modulations of the conditions in the earth's atmosphere.

Acknowledgments. The author is grateful to the staff, at present and in the past, of the Norikura Solar Observatory for their efforts in operating the instruments for over fifty years.

\section{References}

Barnes, J. E. and D. J. Hofmann, Lidar measurements of stratospheric aerosol over Mauna Loa Observatory, Geophys. Res. Lett., 24, 19231926, 1997.

Dutton, E. G., P. Reddy, S. Ryan, and J. J. DeLuisi, Features and effects of aerosol optical depth observed at Mauna Loa, Hawaii: 1982-1992, J. Geophys. Res., 99, 8295-8306, 1994.

Husar, R. B., J. M. Prospero, and L. L. Stowe, Characterization of tropospheric aerosols over the oceans with the NOAA advanced very high resolution radiometer optical thickness operational project, J. Geophys. Res., 102, 16889-16909, 1997.

Ichimoto, K., M. Noguchi, N. Tanaka, K. Kumagai, K. Shinoda, T. Nishino, T. Fukuda, T. Sakurai, and N. Takeyama, A new observing system of the corona at Norikura, Publ. Astron. Soc. Japan, 51, 383-391, 1999.

LaBonte, B., Sky brightness measurements at Haleakala, 1955-1998, IIFASG Preprint 119, 12 pp., Institute for Astronomy, University of Hawaii, 1999.

Lean, J., Solar ultraviolet irradiance variations: A review, J. Geophys. Res., 92, 839-868, 1987.

Long, C. S. and L. L. Stowe, Using the NOAA/AVHRR to study stratospheric aerosol optical thickness following the Mt. Pitatubo eruption, $J$. Geophys. Res., 21, 2215-2218, 1994.

Lyot, M. B., A study of the solar corona and prominences without eclipses, Monthly Notices Roy. Astron. Soc., 99, 579-595, 1939.

Percival, D. B. and A. T. Walden, Spectral Analysis for Physical Applications, pp. 489-496, Cambridge University Press, Cambridge, 1993.

Sakurai, T., M. Irie, H. Imai, H. Miyazaki, and J. Sykora, Emission line intensities of the solar corona and sky brightness observed at Norikura: 1950-1997, Publ. Natl. Astron. Obs. Japan, 5, 121-137, 1999.

Sato, M., J. E. Hansen, M. P. McCormick, and J. B. Pollack, Stratospheric aerosol optical depths, 1850-1990, J. Geophys. Res., 98, 22987-22994, 1993.

Thompson, D. T. and G. W. Lockwood, Atmospheric transparency at Flagstaff, Arizona, 1972-1996: Baselines and volcanic episodes compared, Geophys. Res. Lett., 23, 3349-3352, 1996.

Ulrych, T. J. and T. N. Bishop, Maximum entropy spectral analysis and autoregressive decomposition, Rev. Geophys. Space Phys., 13, 183-200, 1975 .

van de Hulst, H. C., The chromosphere and the corona, in The Sun, edited by G. P. Kuiper, pp. 207-321, The University of Chicago Press, 1953.

T. Sakurai (e-mail: sakurai@solar.mtk.nao.ac.jp) 